\title{
Research on Recording Methods of Eye Tracking Technology Based on Human Engineering
}

\author{
Chen, Leyang ${ }^{1, a}$, Yang, $\mathrm{Li}^{2, \mathrm{~b}}$, and Hu Minghui ${ }^{3, \mathrm{c}}$ \\ ${ }^{1}$ Beijing Institute of Graphic Communication, Beijing 102600, China \\ ${ }^{2}$ Beijing Institute of Graphic Communication, Beijing 102600, China \\ ${ }^{3}$ Beijing Institute of Graphic Communication, Beijing 102600, China \\ a34373522@qq.com, b191889587@qq.com, ${ }^{c} 184639923 @ q q . c o m$
}

Keywords: Eye Tracking Technology, Recording Method of Eye Movement Device

Abstract. Eye Tracking is a technology which can accurately tracking the path of human vision, by recording some key data of human eye movement process. The difference in the recording methods selection will decide accuracy and subjectivity of eye movement data. The device to record the date is called Eye Tracking System.

\section{Introduction}

In as early as $19^{\text {th }}$ century, people started to study people's psychological activity by observing eye movement, to study the relation between eye movement and human psychological activity by analyzing the recorded eye movement data. The appearance of eye tracking device provided a new effective tool to use eye movement technique to explore visual information process mechanism in different conditions, and to observe its direct or indirect, interesting and marvelous relation with psychological activity. Eye movement technique has gone through the development from Visual Observation, Afterwards Visual, Mechanical recording, Optics recording ${ }^{[1]}$, Video recording and many other solutions. Eye movement technique is to study the inherent cognition process by extracting data like focus points, focus time and frequency, jumping distance, pupil size from eye movement tracking record. After 1960s, with development of video, infrared and microchip technique, especially the application of computer technology, the high accuracy eye tracking devices has been developed rapidly. The improvement has greatly promoted the eye movement study application in different areas.

\section{Categories of Eye Movement Recording}

Eye movement device is a device to record the eye movement data. It has developed from interfering to non-interfering.

\section{Realization Methods of Interfering Eye Movement Recording}

Realization of interfering eye movement recording methods mainly includes Visual Observation, Mechanical Recording, Current Recording and Electromagnetic Recording. ${ }^{[2]}$ It was developed in a period with low computer process ability

Visual observation is to use human eye and simple recording device to directly observe objects' eye movement. This method is simple and easy manipulate. But because it observed by human eye, so the accuracy of the test is very low.

Mechanical recording is to tracking eye movement by using mechanism to connect human eye and test device. Mechanical Recording ${ }^{[3]}$ is complicated, difficult to manipulate, low accuracy and greatly interfere objects.

The basic principle of Current Recording is based on the electric potential between cornea and retina in eye movement. By importing current to the equipment on the skin near eyes, it can record current change caused by eye movement. Eye Movement can be shown by galvanometer or 
oscillograph. Current recording is a relatively high accuracy tracking technology in that period, but as the mechanical recording solution, current recording has big influence on objects.

Contact Lens Method is to record eye movement by measuring the induction voltage caused by eye movement, with invisible lens with special coil attached to eyes ${ }^{[41]}$. Contact lens is the most accurate solution in interfering ones. But it needs to anesthetize human eyes and attach equipment on it, so it creates the most interference to subject's eye and can causes certain physiological harm.

\section{Realization of Non-interfering Eye Movement Recording}

Realization methods of eye movement recording mainly include Cornea Reflection Method, Sclera-Iris Edge Method and Pupil-Cornea Reflection Vector Method

Cornea Reflection Method: Cornea Reflection light is reflection of the light irradiates on cornea. The light will create a light spot after cornea reflection, called cornea reflection spot. In human eye, cornea protruded from eyeball surface. So when eyes move, light will incident on cornea in different angles, the position of cornea reflection spot will change as well. By recording eye movement image with eye camera, to the change of cornea reflection spots, we can obtain the position of virtual image by using real time image processing technique ${ }^{[5]}$. In this way, we can track vision movement. This method is mainly used on eye movement mechanics and focus calibration, but the error of the head is relatively huge.

Sclera-Iris Edge Method: This method used infrared light to irradiate on human eyes, and then use two infrared light sensitive tubes to absorb the reflected infrared light from the edge of Sclera edge and iris edge. Infrared light absorbed will change as the movement of eyes. When eye come to one side, iris will move to this side, and the infrared light absorbed by light sensitive tube will decrease; and the sclera reflection on the other side will increase ${ }^{[6]}$, making the infrared light absorbed by the other side light sensitive tube increased. By using the differences of this two signal, the movement of eye is measured without contact. This method is applied in eye mechanics and focus calibration. Its horizontal accuracy is high, but vertical accuracy is low, interfere is huge and the head error is huge.

Pupil-Cornea Reflection Vector Method. Firstly, capturing eye image by eye camera, and then get pupil center point by image processing. Afterwards, to use cornea reflection point as base point of relative position of eye camera and eyeballs. With the vision vector coordinate obtained by pupil center position, eye focus is calibrated. This method is commonly used in focus calibration. It has high accuracy, low interference and low head error. This is the most widely used method of nowadays eye tracking device.

\section{Types and System Constitution of Eye Movement Device}

First type is helmet eye movement device. Infrared light, eye camera and field of view camera are mounted on helmet. Eye image collected is analyzed by data analyzing software in computer. Afterwards, by pinpoint the calibrated point on the image of field of view camera with calibration calculation, the calibration of human eye focus is done. Helmet eye movement device allows certain degree free movement of object's body and head.

Second type is desktop eye movement device. Objects of such device don't need to ware any instrument. Camera will capture object's eye movement after adjustment ${ }^{[7]}$. It only allows users head moved in little room and doesn't allow any movement of body. For example, there is a kind of base type eye movement device. It's like sight test device. Object's forehead and chin stick to the instrument. The software process is the same as helmet type.

Modern eye movement device consists of 4 system, including optics system, pupil center coordinate extracting system, field of view and pupil coordinate superposition system and data recording and analysis system. There are 3 types of eye movement: fixation, saccades and pursuit movement. 


\section{Summary}

Recent test standard is mostly outside characteristics of eye movement. It lacks study on human's cognition characteristic in decision process. It's another important trend in eye movement study to investigate inside process of human selection and judgment. Eye movement data is a observing data in behavior analysis which show exterior characteristic of eye movement. If it can combines with cognition data and reflects interior decision characteristic of human in fast response situation, the study will go deeper.

\section{Acknowledgements}

This work was financially supported by the Institute Level Projects Funded by KM201310015009,

Beijing Municipal Education Commission tech surface project.

\section{References}

[1] Shupert C, Fuchs A F. Development of Conjugate Human Eye Movement [J ] . Vision Res, 1988, 28(5): $585 \sim 596$.

[2] Daxsibm H. The Eye [M] . Academic Press, New York and London: Vol 3. 1963.

[3] Gauthier G M. Vertical and horizontal Eye Movement Recording in the Unrestrained Cat [J] . IEEE Trans Biomed Eng, 1975, BME-22(3): 202-207.

[4] Jones R. Two Dimensional eye Movement Recording Using a Photoelectric Matric Method

[5] Information on http://www.learning-systems.ch/multimedia/eye\%20movements\%20problem\%20solving.swf

[6] Information on http://www.learning-systems.ch/multimedia/forsch1e.htm

[7] You Jin Ko,Eui Chu Lee,Kang Ryoung Park.A robust gaze detection method by compensating for facial movements based on corneal specularities .Pattern Recognition Letters.

2008,7,29(10).1474-1485. 